\title{
Percepción de formación en entrevista, relación y comunicación médico paciente. Encuesta en médicos graduados
}

\author{
Cecilia Sogi ${ }^{1}$, Salomón Zavala ${ }^{1}$, Miguel Ángel Oliveros ${ }^{1}$, Carlos Salcedo ${ }^{2}$
}

Resumen

Palabras clave

\begin{abstract}
Objetivo: Identificar factores relacionados con habilidades de entrevista, relación y comunicación médico paciente. Diseño: Estudio tipo encuesta. Lugar: Facultad de Medicina, Universidad Nacional Mayor de San Marcos. Participantes: Médicos graduados de medicina. Métodos: Se utilizó una base de datos de encuesta a graduados de medicina sobre su formación en habilidades clínicas básicas. Principales medidas de resultados: Habilidades clínicas en entrevista, relación médico paciente y comunicación. Resultados: Los datos fueron obtenidos de 917 graduados, $59 \%$ varones, $56 \%$ entre los 25 y 29 años de edad. La cuarta parte la constituyó médicos graduados de la Universidad Nacional Mayor de San Marcos, la tercera parte, graduados en el año 2003; el 85\% respondió haber tenido formación en las habilidades en mención. El puntaje en habilidades se asoció a tener formación en las mismas en el análisis bi y multivariado y ser graduado antes del 2003, en el análisis multivariado. El comentario de los encuestados apuntó hacia la importancia de evaluar la formación en habilidades clínicas. Conclusiones: El ejercicio profesional requiere de formación en las habilidades clínicas básicas de años de experiencia clínica en opinión de los graduados.
\end{abstract}

Relación médico-paciente; entrevistas; comunicación; educación médica.
Perception of education in medical interview, physician patient relationship and communication skills. Survey of medical graduates

Abstract

Objective. To identify factors associated to medical interview, physician-patientrelationship and communication skills of medical graduates Design: Surveytype study. Setting: Faculty of Medicine, Universidad Nacional Mayor de San Marcos. Participants: Medical graduates. Methods: Data base of a survey to medical graduates on their training in core clinical skills was used. Main outcome measures: Medical interview, physician patientrelatioship and communication skills. Results: The data base was obtained from 917 graduates, $59 \%$ male, $56 \%$ between 25 -29years old. A quarter was graduated from the Universidad Nacional Mayor de San Marcos, one third graduated in 2003; 85\% responded that they had been trained in core clinical skills. The highest score in clinical skills was related to have had training in those skills in bi and multivariate analysis and to be graduated before 2003, in multivariate analysis. Two third of the comments showed positive attitudes towards this type of study. Conclusions: Clinical practice

1 Instituto de Ética en Salud. Facultad de Medicina, UNMSM. Lima, Perú. 2 Hospital Nacional Daniel Alcides Carrión. Callao, Perú. requires training in clinical skills likewise years of clinical experience, according to the perception of the graduates.

Key words: Physician-patient relationship; interviews; communication; education, medical.

\section{INTRODUCCIÓN}

El proceso de acreditación de las escuelas de medicina exige que los cursos que integra el currículo de estudios sean elaborados en base de competencias. Es decir que, además de los conocimientos, considere las habilidades, destrezas y actitudes necesarias para el ejercicio profesional.

En nuestro medio, tradicionalmente, la evaluación del estudiante de medicina en el pregrado se limita, por lo general, al campo de los conocimientos, siendo el método más utilizado el examen escrito objetivo, de elección múltiple. Así, son pocas las 
oportunidades en que se evalúa la competencia clínica.

La competencia profesional es, en la definición de Epstein y Hundert, "el uso habitual y juicioso de conocimiento y habilidades técnicas y de comunicación, razonamiento clínico, emociones, valores y reflexiones en la práctica diaria en beneficio del individuo y de la comunidad a servir" ${ }^{1}$ ).

En otras palabras, Carr señala que la competencia se basa en habilidades clínicas básicas, conocimiento científico, y desarrollo moral. Incluye una función cognitiva adquiriendo y utilizando conocimiento para resolver problemas reales de la vida, una función de integración -utilizando datos biomédicos y psicosociales en el razonamiento clínico, una función de relación comunicándose efectivamente con pacientes y colegas; y una función afectiva/moral -la voluntad, paciencia, y sensibilidad emocional para utilizar estas habilidades juiciosa y humanamente $\left({ }^{2}\right)$.

Ambas definiciones corresponden, según Holmboe y Hawkins, a una definición amplia de competencia, que incorpora los dominios de conocimiento, habilidades y actitudes. A diferencia de otros educadores que la definen más estrechamente, entre ellos Miller, quien divide las habilidades clínicas en una pirámide de cuatro niveles: conocimiento (sabe), competencia (sabe cómo), destreza (demuestra cómo) y acto (hace) $\left.{ }^{3}\right)$.

En los Estados Unidos, el Accreditation Council for Graduate Medical Examination (citado por Epstein y Hundert) definió seis áreas de competencia y algunas formas de evaluarlas: atención del paciente (incluyendo razonamiento clínico), conocimiento médico, aprendizaje y mejora sobre la base de la práctica (incluyendo manejo de la información), habilidades de comunicación y relación interpersonal, profesionalismo y prác- tica basada en el sistema (incluyendo economía de la salud y trabajo en equipo) $\left({ }^{1}\right)$.

En el Reino Unido, estudios que evalúan conocimientos y actitudes hacia la guía de la 'Buena Práctica Médica' demuestran que, aunque los médicos la conocen y muchos están en proceso de cambiar su propia práctica, en la medida que uno se mueve hacia la parte de deberes que tienen que hacer más con lo interpersonal, en oposición a los aspectos técnicos de la medicina, solo unos pocos creen que el pobre desempeño en ello amerite sanciones $\left({ }^{4}\right)$. Otra encuesta de médicos en el Reino Unido halló que muchos ven las deficiencias en el área de habilidades interpersonales y actitudes como menos serios que las fallas en procedimientos técnicos. Pese a que el patrón de quejas en los hospitales y la práctica general son preocupaciones de pacientes por deficiencias en modales, actitudes y comunicación del médico $\left(^{5}\right)$.

En los Estados Unidos, Kassebaum y Eaglen, obtuvieron información sobre los métodos utilizados para evaluar logro de estudiantes en cursos y prácticas clínicas, de 97 escuelas de medicina que respondieron a encuestas de acreditación del Liaison Committee on Medical Education (LCME), entre julio de 1993 y junio de 1998. Los autores hallaron que 50 a $70 \%$ del grado académico alcanzado por el estudiante está basado en evaluaciones que hacen los docentes y residentes de las presentaciones y discusiones de caso, escasamente relacionados con habilidades de relación interpersonal, buena relación con pacientes, historia clínica lógica y secuencial y examen físico. El número de escuelas de medicina que utilizan pacientes estandarizados aumentó, entre 1993 y 1998, de $34,1 \%$ a $50,4 \%$, sin embargo, ellas aún fallan en emplear métodos de evaluación de logro de habilidades y conductas que los estudiantes necesitan aprender para la práctica médica $\left(^{6}\right)$. 
En 1991, Novack y colaboradores llevaron a cabo una encuesta sobre enseñanza de habilidades en entrevista y relación interpersonal en escuelas de medicina norteamericanas. Los autores concluyen que ahora prácticamente todas las escuelas ofrecen enseñanza en las mencionadas habilidades, sin embargo, existen grandes diferencias entre ellas en la calidad y la frecuencia de tales cursos $\left({ }^{7}\right)$.

Respecto a habilidades de comunicación, Ashbury y colaboradores llevaron a cabo una encuesta en médicos generales y de familia en Newfoundland. La mayoría de encuestados $(83,8 \%)$ señaló que sus habilidades de comunicación son tan importantes como las técnicas para alcanzar resultados positivos en la evolución del paciente. Un tercio a la mitad calificaron de inadecuada la formación en tales habilidades. Señalaron también la necesidad de mejorar en el uso de 8 de las 13 estrategias de comunicación específicas en el encuentro con el paciente $\left({ }^{8}\right)$.

\section{MATERIALES Y MÉTODOS}

Los datos del presente estudio corresponden a la encuesta de habilidades clínicas en entrevista, relación médico paciente y comunicación $\left({ }^{9}\right)$. En esta oportunidad se presenta los resultados del análisis bivariado y multivariado, siendo la variable dependiente puntaje en habilidades clínicas y las variables independientes sexo, edad, año de graduación, universidad de procedencia y tener formación en las habilidades en mención.

La población de estudio estuvo conformada por médicos graduados, postulantes a las plazas de residencia de la Universidad Nacional Mayor de San Marcos (UNMSM). El total de inscritos en el proceso de admisión 2005 fueron 1410 y completaron el cuestionario de habilidades clínicas $947(67 \%)$.
El cuestionario utilizado evalúa: inicio de la sesión de entrevista, obtiene información, información al paciente, comprensión del paciente, habilidades de relación, modales del médico y término de la entrevista. La validez de contenido se determinó mediante la prueba de jueces y la confiabilidad mediante el coeficiente alfa de Cronbach $\left({ }^{9}\right)$.

Se solicitó autorización para la aplicación del cuestionario a la Dirección de la Unidad de Postgrado, Facultad de Medicina de la UNMSM así como a la Comisión a cargo del proceso de admisión.

Se recogió los datos en el proceso de inscripción de los postulantes, el mismo que tuvo lugar en marzo del 2005. Dos personas entrenadas estuvieron a cargo del trabajo de campo, supervisadas por los investigadores.

Concluido el trabajo de campo, los cuestionarios fueron revisados, separándose aquellos incompletos, los que marcaron todas las respuestas en una sola columna y los que respondieron algunas veces en la pregunta 53 ¿He respondido sincera y honestamente a las frases anteriores? En este proceso se separó 30 cuestionarios (3\% de 947), los mismos que fueron descartados, quedando 917 en la base de datos.

En cada cuestionario se sumó las respuestas (1-5) de las 52 preguntas, obteniéndose un puntaje total (en habilidades clínicas), el mismo que fue utilizado para fines del análisis bi y multivariado.

En lo que respecta a ética, el consentimiento fue verbal, luego de explicar que la participación era voluntaria y que los datos serían utilizados solo para los propósitos de la investigación.

El procesamiento y análisis de datos se realizó usando los paquetes estadísticos SPSS 9,0 para Windows y EpiInfo 6,0 para DOS. Elaborada la base de datos en Excel, 
se procedió a hacer tablas con una y dos variables. En el análisis de datos cualitativos se utilizó el Atlas ti.

Se aplicó chi cuadrado en las tablas bivariadas, como prueba de independencia. Para la prueba de significado estadístico, se utilizó alfa $=0,05$ o $p<0,05$.

Se utilizó análisis de regresión logística para determinar la asociación entre variables independientes (sexo, edad, año de graduación, universidad de procedencia, formación en habilidades clínicas) con la variable dependiente (puntaje total en habilidades). Para ello, se dicotomizó la variable dependiente en: 0 para puntajes mayores que la media y 1 para puntajes menores o iguales que la media

\section{RESULTADOS}

Participaron en el estudio las dos terceras partes de los postulantes, inscritos en el proceso de admisión 2005 a plazas de residencia en la UNMSM. Los no participantes fueron, en su mayoría, personas que mediante poder inscribieron al postulante y por consiguiente no se les administró el cuestionario.

Del total de participantes en el estudio $(\mathrm{n}=917), 58,8 \%$ correspondió a varones y más de la mitad $(55,8 \%)$ estuvo en el grupo de edad entre 25 y 29 años, seguidos de aquellos que estaban entre 30 y 34 años (31\%). La edad media de los varones fue 30,3 (DE 4,8) y de las mujeres 29,0 (DE $3,7)$; la edad mínima fue 23 años y la máxima 66 .

Los participantes eran egresados de 20 facultades de medicina del país y nueve del extranjero (Argentina, Bolivia, Ecuador, México, España y Rusia). La cuarta parte de los médicos graduados $(24,8 \%)$ fue de la UNMSM, le seguían los de la Universidad Nacional San Luis Gonzaga de Ica
(12\%) y de la Universidad Particular San Martín de Porras (11,8\%).

La tercera parte de los postulantes se graduó en el año 2003 (31\%), seguidos de los graduados en el $2004(20,8 \%)$.

El $84,5 \%$ de los participantes respondió haber tenido formación en las citadas habilidades en diversos cursos: ética, medicina, medicina comunitaria, psicología y psiquiatría, especialidades médicas y quirúrgicas, y otras, como comunicación, metodología de investigación, administración, gerencia, filosofía, historia, antropología, enfermería, ciencias básicas. Varios mencionaron haber tenido formación en habilidades clínicas en todos o la mayoría de cursos durante la carrera.

Adicionalmente a la amplia variedad de cursos mencionados, la formación en las habilidades que nos ocupa mostró diversas deficiencias en el análisis univariado $\left({ }^{9}\right)$.

Con relación al análisis bivariado, el puntaje total en habilidades clínicas se asoció significativamente a la formación en dichas habilidades $(p<0,002)$ y al haber hecho estudios de pregrado en Lima $(p<0,01)$.

Y, con relación al análisis multivariado, la Tabla 1 presenta los resultados del análisis de regresión logística. Se observa que el puntaje total en habilidades clínicas se asoció significativamente al año de graduación (antes del 2003) y la formación en las mismas.

El puntaje en habilidades clínicas se asoció significativamente a la formación en dichas habilidades, tanto en el análisis bivariado como en el multivariado; y al año de graduación antes del año 2003, en el análisis multivariado.

Uno de cada cinco encuestados $(21,3 \%$ de 917) hizo comentarios. Éstos fueron clasificados en cinco categorías, relacionados con: educación médica, método del presente 
Tabla 1. Análisis multivariado.

\begin{tabular}{lccc}
\hline \multirow{2}{*}{ Variable } & \multicolumn{3}{c}{ Regresión logística } \\
\cline { 2 - 4 } Edad & beta & DE & Sig \\
Sexo & 0,1340 & 0,1534 & 0,3825 \\
Estudio pregrado & 0,2273 & 0,1360 & 0,0948 \\
Año de graduación & 0,3778 & 0,1738 & 0,0297 \\
Formación & 0,4844 & 0,1877 & 0,0098 \\
Constante & $-1,1946$ & 0,3306 & 0,0003 \\
\hline
\end{tabular}

DE: Desviación estándar.

Sig: Prueba de significancia estadística.

estudio, actitud hacia la encuesta, otro y tiempo. A continuación se presenta ejemplos de estas categorías, a excepción de la última, presentada en artículo previo:

Actitud hacia la encuesta. Casi la cuarta parte $(23,6 \%)$ de todos los comentarios, expresó una actitud positiva hacia el cuestionario/estudio. Unos lo consideraron una autoevaluación necesaria; otros calificaron el cuestionario de excelente, interesante, importante, oportuno, útil y varios solicitaron la publicación de los resultados.

- Considero importante la ejecución de este test que nos permite la autoevaluación y corrección.

- Nos lleva a ser más reflexivos en cuanto al cuidado del paciente y ser más ético y moral.

- Es interesante conocer a través de este test que hay muchas cosas que mejorar y definitivamente todo es importante. Espero saber los resultados.

- Agradecer por el interés en que el médico mantenga viva su formación humana y técnica, que redunda en beneficio del paciente y de la satisfacción espiritual del médico que se renueva y realiza en cada acto médico.

- Me parece muy oportuna la realización de esta encuesta para poder contribuir al mejoramiento de la calidad de atención que se brinda a los pacientes.

- Felicito a la Facultad por este tipo de encuesta, para mejorar la atención integral, oportuna y de calidad.

- Los resultados de la encuesta se debe publicar en internet.

- Sería interesante ver los resultados de este cuestionario publicado en la página Web del MINSA, COMP, IDREH y otros similares.
Educación médica. El 11,8\% del total de comentarios se refiere a la necesidad de una formación en la relación médico paciente, y otros se refieren a algunas características de la formación en las habilidades en mención.

- Muchos profesionales en salud han perdido el concepto de la relación médico paciente; por lo tanto, es a este nivel donde se tiene que actuar, ya que es lo principal para la satisfacción final de la atención médica.

- El médico debe ser formado paralelamente en lo humanístico y lo académico.

- Es muy importante tratar este aspecto, puesto que la relación médico paciente es no sólo técnica; requiere experiencia y guía.

- En el curso de la carrera, se incide en gran medida en el tratamiento en hospitalización y emergencia, pero no en consulta ambulatoria, la cual presenta diferencias pequeñas pero importantes de reconocer.

- Además de los cursos de semiología y propedéutica que normalmente se dicta, se debe dar cursos de gramática y redacción, relaciones interpersonales y de ética y deontología.

- Relación médico paciente no se aprende en un curso, eso viene de la formación familiar: la sensibilidad, la percepción de problemas y los deseos de ayudar al prójimo.

- No solo con tratamiento farmacológico se trata al paciente, sino con palabras de alivio.

- Pero, las universidades no enseñan alguna de estas habilidades, más por el contrario, se observa un maltrato a los pacientes, especialmente si son de condición humilde, y se debería dar más énfasis en ética médica.

- Casi nadie trata pacientes; nos educan a tratar enfermedades y cada uno somos un mundo distinto.

Otros. E1 28,2\% de todos los comentarios fue clasificado como otros. Algunos enfatizaron la importancia del tema de estudio, tal como lo expresan los siguientes ejemplos:

- Permitir una buena relación médico paciente, con honestidad, son factores muy importantes para el éxito del tratamiento del paciente.

- Es muy importante la habilidad clínica del galeno en la entrevista médico paciente, para un diagnóstico certero y un mejor conocimiento del paciente.

- El trato cordial, amable, con un verdadero interés por los problemas de salud del paciente, es la base para un tratamiento eficaz. 
- El respeto y la comunicación con el paciente siempre dará como resultado una buena relación médico paciente.

- El trato, saber escuchar y la confianza que se da al paciente repercute directamente en el éxito o fracaso de su tratamiento.

Otros dieron razones de por qué es importante el tema

- No hay médico sin paciente.

- Esta encuesta nos recuerda lo importante que es ver a nuestros pacientes como personas y no solo como enfermos y enfermedad.

- Es importante evaluar al paciente no solo en su problemática física sino emocional y sobre su entorno, puesto que las soluciones no dependen únicamente de cuánto sabemos como médicos, sino en cómo lo atendemos, con dedicación y dándole confianza.

- Se trata de mejorar la calidad de los servicios de salud.

Uno mencionó cómo debe ser la relación médico paciente.

- Sería bueno e interesante, coordinados paciente y médico, visión horizontal, ver al paciente como amigo.

Otros comentaron que el médico no actúa según la formación y el poco uso de la comunicación no verbal.

- Muchas veces, el médico conoce y sabe cómo debe ser el trato y la entrevista con un paciente; pero, son pocos los que realmente aplican lo aprendido, y tienen una identificación pobre con el paciente.

- Muchos médicos aún no logran entender lo importante que es para todo paciente mostrarles una sonrisa sincera.

Finalmente, uno de los comentarios es un ejemplo claro de una formación netamente biomédica.

- Creo que lo más importante de todo médico es diagnosticar la enfermedad, casi siempre debido a que muchas veces esto no se da y se trata una enfermedad. Por otra parte, falta de conocimiento médico, trayendo consigo la muerte del paciente; es necesario siempre saber que uno tiene que saber todo sobre la medicina, porque siempre nos enfrentamos a patología o anormalidades y hay que ganarles a éstas y siempre prevenirlas; los demás detalles son superficiales.
Método de estudio. El $23 \%$ de todos los comentarios hizo críticas al método de estudio:

- El planteamiento de las preguntas es algo sugerente o inducen a la respuesta. Falta mayor objetividad en las preguntas.

- Es difícil hablar de siempre y nunca, recomendaría que las opciones fueran nunca o casi nunca, pocas veces, algunas veces.

- Parece que las preguntas son directas, con tendencia a ser todas positivas.

- Muchas preguntas para una sola encuesta.

- Con toda franqueza, dudo mucho que una simplificación tan abusiva y aberrante del acto médico (al estilo de las competencias) sirva realmente para evaluar la calidad de atención del profesional, menos aún para normarla.

Algunos mencionaron a quiénes se debe administrar el cuestionario:

- Hagan con el personal administrativo. Este cuestionario debería ser dado a pacientes para la evaluación de sus médicos. La relación médico paciente debe incluir cursos desde el 1er año de estudios. Sería bueno aplicarlo a los estudiantes de 6to año; es internado, donde la relación médico paciente es importante. Este curso debería darse en los colegas que iniciarán el SERUMS.

Otros sugirieron el método de la observación participante:

- Esta encuesta debería trasladarse al consultorio externo de nuestros EESS, no como preguntas sino con un testigo observador; así, lograría acercarse en el campo mismo de la realidad; empero, la presente es un inicio de mejora a cambio. ¡Muy bien $i$

- Verificar los resultados haciendo visitas de incógnito a los distintos consultorios.

Varios comentarios hicieron mención al contexto de la relación clínica:

- Por mi situación de atención en la salud pública en la zona andina, muchas veces limito mi oportunidad de brindar una atención completa de calidad por la dificultad del idioma (quechua).

- Por el lugar donde actualmente trabajo (rural), es posible tomarse algo más del tiempo establecido para cada 
consulta; tiempo que ayuda a compenetrarnos un poco más con la problemática del mismo; lo cual sería muy recomendable se hiciera en todas las instituciones de salud.

- La información médica debe ser evaluada según el lugar de trabajo del médico. Como sabemos en las instituciones públicas el trato es pésimo, lo que no es en la privada. Esto condiciona al médico.

\section{DISCUSIÓN}

Limitaciones del estudio. La principal limitación del estudio es el método utilizado, pues se trata de una encuesta autoadministrada que sobreestima el uso real de habilidades $\left({ }^{8}\right)$; asimismo, el cuestionario es una adaptación de tres instrumentos y, como tal, nuestros hallazgos no son estrictamente comparables.

No obstante las limitaciones señaladas, nuestros datos se obtuvo de 917 graduados de medicina, de 20 universidades públicas y privadas del país y 9 del exterior.

Formación en habilidades clínicas. En nuestro estudio, el análisis bi y multivariado mostró asociación significativa entre puntaje en habilidades clínicas y tener formación en las mismas. Este resultado, aunque esperado, no señala las deficiencias cualitativas de ese aprendizaje. En publicación previa, comunicamos que pese a que el $85 \%$ de los encuestados respondió haber llevado cursos en las habilidades que nos ocupa, se identificó deficiencias en: método clínico, obtención de información psicosocial, evaluación de la competencia en la información al paciente, comunicación no verbal, manejo de emociones y término del encuentro con el paciente $\left({ }^{9}\right)$.

Nuestro hallazgo de deficiencias en el aprendizaje de las habilidades clínicas estaría reflejando lo que ocurre con su enseñanza en nuestras universidades, similar a lo que hallaron Corbett y Whitcomb en las escuelas de medicina norteamericanas, que la formación en habilidades clínicas varía mucho y que no existe un plan de estudios estándar. Comentan los autores que muy pocas parecen aproximarse a tal formación como un proceso de desarrollo explícito a lo largo de los cuatro años de estudios. Solamente una minoría tiene objetivos explícitos que guían la educación de los estudiantes, los mismos que varían entre escuelas. La mayoría proporciona alguna educación formal en habilidades clínicas, primariamente durante el primer y segundo año de estudios. El grado en que las disciplinas prácticas participan de manera organizada en la enseñanza y evaluación de las mencionadas habilidades varía substancialmente. Se sigue asumiendo que, en el internado, los estudiantes adquieren las habilidades necesarias para la formación posgraduada, pero la mayoría de las escuelas no la verifican $\left({ }^{10}\right)$.

Respecto a la evaluación del aprendizaje, nuestras universidades continúan utilizando el examen escrito. En las escuelas de medicina norteamericanas, Corbett y Whitcomb utilizan pacientes estandarizados (PE) para evaluar ciertas habilidades clínicas, en diferentes momentos, durante los estudios clínicos. En la mayoría se exige que los estudiantes aprueben un examen que incluye evaluación de habilidades clínicas seleccionadas antes de la graduación. En la última década, el número de escuelas que utilizan $\mathrm{PE}$ en la evaluación final aumentó de 26 a 67, sin embargo, el examen escrito para evaluar esas habilidades persiste en la quinta parte de las escuelas. Muy pocas $(n=25)$ evalúan el desempeño del estudiante a través de la observación directa del docente $\left({ }^{10}\right)$.

Experiencia clínica. El puntaje en habilidades clínicas se asoció a ser graduado antes del año 2003. Esto indicaría una mayor experiencia clínica o años de ejercicio profesional, hallazgo que coincide con la afirmación de Moreno Rodríguez, que: "alcanzar una elevada maestría en el interrogato- 
rio requiere atender muchos pacientes, pero también un número de años” $\left({ }^{11}\right)$.

Evaluación de habilidades clínicas. En nuestro estudio, el comentario de los encuestados apunta hacia la necesidad de una mejor enseñanza y evaluación de las habilidades clínicas.

Respecto a los métodos para evaluar la competencia clínica, Holmboe y Hawkins llevaron a cabo una revisión y concluyen que un programa de competencia debe incluir instrumentos que midan conocimiento, habilidades y actitudes e incorporar la observación directa. Si se usa eficazmente, la evaluación en servicio (conocimiento), las escalas (habilidades y actitudes) y el ejercicio de evaluación clínica (habilidades) pueden servir como núcleo de un programa exitoso. Sin embargo, la confiabilidad y precisión de la evaluación docente usando estas medidas han demostrado consistentemente ser subóptimas. Los programas pueden necesitar hacer uso de técnicas, como encuentro con paciente estandarizado (para evaluar mejor habilidades y actitudes), o auditoría de historias clínicas (para examinar más efectivamente la práctica clínica) $\left({ }^{3}\right)$.

La evaluación de las habilidades es una tarea compleja. Albanese hace una revisión de los factores vinculados con la erosión de los estándares profesionales. Dice el autor que las evaluaciones tienen sesgos positivos (error de generosidad), discrimina poco $y$ a menudo falla en documentar serias deficiencias. Las potenciales fuentes de estos problemas se relacionan con los mecanismos de la tarea de evaluación, el sistema utilizado para la calificación y los factores que afectan el juicio del examinador $\left({ }^{12}\right)$.

Otro tema que surgió en comentarios de los participantes fue la necesidad de autoevaluación. La autonomía profesional va de la mano con la autorregulación, y ésta implica la evaluación de la práctica médica. Al respecto, Gordon señala que un objetivo en la formación de profesionales de la salud debe ser enseñar a que éstos se autoevalúen. En su revisión del tema halló que, en aprendices adultos, la autoevaluación está íntimamente ligada al autoconocimiento o atribuciones de habilidad estables, al punto que su opinión acerca de la misma parece ser ordinariamente refractaria, tanto ante la evidencia objetiva como al juicio de los evaluadores calificados... Adicionalmente, halló que los estudiantes pueden aprender gradualmente a aplicar los estándares y criterios de la profesión a sus pares, aunque permanecen incapaces de evaluarse ellos mismos, como lo hacen con los demás $\left({ }^{13}\right)$.

Respecto a la enseñanza de habilidades de comunicación, Kidd y colaboradores plantean que debe ir de la mano con la formación en habilidades clínicas. Dicen los autores que la dicotomía no refleja la experiencia clínica y que puede llevar a la formación de médicos no aptos; de clínicos con adecuado conocimiento clínico, pero evaluados como incapaces de aplicar sus habilidades en una atención clínica efectiva. La evidencia de ensayos aleatorios controlados señala que la educación médica interactiva continuada es efectiva en cambiar positivamente el ejercicio clínico y ha estimulado el desarrollo de otros modelos de aprendizaje integrado $\left({ }^{14}\right)$.

Respecto a la formación en habilidades de comunicación y su efecto en la satisfacción del paciente, Brown y colaboradores, después de un estudio aleatorio controlado, concluyen que, para mejorar la satisfacción global del paciente, los programas de formación en habilidades de comunicación tendrían que ser más largos e intensivos, que incluya la enseñanza de un amplio rango de habilidades y que proporcione una retroalimentación continuado sobre el desempeño $\left({ }^{15}\right)$. 
Estudio de Investigación 2005 Con Asignación a la Investigación y Con Incentivo al Investigador.

\section{REFERENCIAS BIBLIOGRÁFICAS}

1. Epstein RM, Hundert EM. Defining and assessing professional competence. JAMA. 2002; 287:226-35.

2. Carr SJ. Assessing clinical competency in medical senior house officers: how and why should we do it? Postgrad Med J. 2004;80:63-6.

3. Holmboe ES, Hawkins RE. Methods for evaluating the clinical competence of residents in internal medicine: A review. Ann Intern Med. 1998;129(1):42-8.

4. McManus IC, Gordon D, Winder BC. Duties of a doctor: UK doctors and good medical practice. Qual Health Care. 2000;9:14-22.

5. Hutchinson A, Williams M, Meadows K, Barbour RS, Jones R. Perceptions of good medical practice in the NHS: a survey of senior health professionals. Qual Health Care. 1999;8:213-8.

6. Kassebaum DG, Eaglen RH. Shortcoming in the evaluation of students' clinical skills and behavior in medical school. Acad Med. 1999;74:841-9.

7. Novack DH, Volk G, Drossman DA, Lipkin M Jr. Medical interviewing and interpersonal skills teaching in US Medical Schools. Progress, problems and promise. JAMA. 1993; 269:2101-5.

8. Ashbury FD, Iverson DC, Kralj B. Physician communication skills: results of a survey of general/family practitioners in Newfoundland. Med Educ Online [revista en Internet]. 2001;6:1. Disponible en: http://www.msu.edu/ dsolomon/ /res00014.pdf

9. Sogi C, Zavala S, Oliveros MA, Salcedo C. Autoevaluación de formación en habilidades de entrevista, relación médico paciente y comunicación en médicos graduados. An Fac Med. 2006;67(1):30-7

10. Corbett EC Jr, Whitcomb M. The AAMC Project on the clinical education of medical students clinical skills education. Washington, DC: Association of American Medical College; 2004

11. Moreno-Rodríguez MA. Deficiencias en la entrevista médica. Un aspecto del método clínico. Rev Cubana Med. 2000;39(2):106-14.

12. Albanese M. Rating Educational Quality: Factors in the erosion of professional standards. Acad Med. 1999;74:652-8.

13. Gordon MJ. Review of the validity and accuracy of self assessment in health professions training. Acad Med. 1991;66:762-9.

14. Kidd J, Patel V, Peile E y Carter I. Clinical and communication skills. BMJ. 2005;330:374-6.

15. Brown JB, Boles M, Mullooly JP, Levinson W. Effect of clinician communication skills training on patient satisfaction. A randomized, controlled trial. Ann Intern Med. 1999;131:822-9.

Manuscrito recibido el 07 de junio de 2007 y aceptado para publicación el 28 de agosto de 2007.

Correspondencia:

Cecilia Sogi Uematsu

Instituto de Ética en Salud

Facultad de Medicina, UNMSM.

Av Grau 755. Lima 1, Perú

Correo-e: ceciliasogi@yahoo.com 